\title{
The Interstellar Gas in the Line of Sight to $\epsilon$ Canis Majoris
}

\author{
C. GRY, ${ }^{1}$ L. LEMONON, ${ }^{1}$ A. VIDAL-MADJAR, ${ }^{2}$ M. LEMOINE, ${ }^{2}$ \\ AND R. FERLET ${ }^{2}$ \\ ${ }^{1}$ Laboratoire d'astronomie Spatiale, CNRS, B.P.8, F-13376 Marseille cedex 12, France \\ ${ }^{2}$ Institut d'Astrophysique, CNRS, 98 bis Boulevard Arago, F-75014 Paris, France
}

\begin{abstract}
We analyse Hubble Space Telescope GHRS observations of the interstellar medium in the direction to $\epsilon \mathrm{CMa}$, the strongest EUV source in the sky located $200 \mathrm{pc}$ away in a region deficient in neutral gas. We show that the neutral gas density is the lowest yet measured in a galactic sightline. The line of sight contains three main components among which the Local Cloud, and we derive their column densities, their velocity their temperature and their turbulence velocity. We discuss the ionization of the Local Cloud and we show that we detect the conductive interface between diffuse local cloud and the hot local bubble.
\end{abstract}

We analyse HST GHRS observations of the interstellar medium in the direction to $\epsilon \mathrm{CMa}$, the strongest EUV source in the sky, located in a region deficient in neutral gas in the high resolution Ech B observations. Three weak components are also detected. We derive their heliocentric velocities, their column densities in Fe II, $\mathrm{Mg}$ II, Mg I, Si III and $\mathrm{CIV}$, upper limits for $\mathrm{N}$ I and $\mathrm{H} \mathrm{I}$, as well as the temperature and turbulence velocity of the three main components.

The analysis leads to the following conclusions :

(1) The neutral gas column density is very low indeed : less than $5 \times 10^{17} \mathrm{~cm}^{-2}$. This value is considerably lower than what had been measured before and it puts constraints on the EUV ionizing flux from $\epsilon$ CMa.

(2) Two of the main clouds are identified as the two components observed in the nearby Sirius line of sight, which shows that they lie less than $3 \mathrm{pc}$ away from the Sun. Beyond this distance, the remaining $180 \mathrm{pc}$ line of sight is thus an interstellar region with the lowest neutral mean density yet measured, with less than $2 \times 10^{-4} \mathrm{~cm}^{-3}$.

This confirms that the EUV flux is essentially unattenuated until the nearby clouds surface, which must therefore be much more ionized than the solar environment.

(3) One of the main components is the local interstellar cloud (LIC) in which the Sun is embedded. Its column density and its temperature $(\sim 7000 \mathrm{~K})$ are in agreement with the lines of sight to Sirius and Capella. We derive a high electron density $n_{e}=0.09_{-.07}^{+.23}$ $\mathrm{cm}^{-3}$ implying a high ionization fraction which could reach $75 \%$.

(4) High ionization state elements like Si III and CIV are detected for the LIC and the third main component, showing that a hot phase is associated with these clouds. An extra absorption well described by a hot $\mathrm{H}$ I counterpart for the LIC is also required by the Lyman $\alpha$ profile fitting. We suggest that we are observing the conduction fronts between the clouds and the hot bubble in which they are embedded.

Note: A more complete description of this work is in press in A\&A. 